\title{
Infarto maligno de la arteria cerebral media en una paciente con meningitis bacteriana
}

\author{
Mirta López $\mathbf{G}^{\mathbf{1}}$, Patricio Mellado $\mathbf{T}^{\mathbf{2}}$, Isidro Huete $\mathbf{L}^{\mathbf{3}}$. \\ Malignant middle cerebral artery \\ territory infarct in one patient with \\ bacterial meningitis
}

The mortality of acute bacterial meningitis (BM) has remained stable in the last decades in spite of the use of new antibiotics, probably due to vascular complications. We report a 68-year-old woman with BM who had a malignant infarction of left middle cerebral artery territory 72 hours after admission to the hospital. The patient experienced a bad evolution and died four days after admission. The arterial involvement in patients with BM is explained by vasospasm of large arteries and vasculitis of small arteries. The medical treatment of a malignant middle cerebral artery infarct has a high mortality (Rev Méd Chile 2004; 132: 1217-20).

(Key Words: Infarction, middle cerebral artery, Meningitis, bacterial)

Recibido el 4 de mayo, 2004. Aceptado el 3 de agosto, 2004.

Servicio de Neurología, Hospital San Pablo de Coquimbo. Escuela de Medicina, Universidad Católica del Norte ${ }^{1}$. Departamentos de Neurología ${ }^{2}$ y de Radiología ${ }^{3}$. Facultad de Medicina, Hospital Clínico de la Pontificia Universidad Católica de Chile. Santiago de Chile.

L a meningitis bacteriana aguda (MBA) es una enfermedad grave, que a pesar de los avances en el tratamiento antibiótico, persiste con una alta tasa de mortalidad y de secuelas neurológicas. En los adultos el Streptococcus pneumoniae es el principal agente etiológico, permaneciendo su mortalidad estable durante las últimas décadas, en alrededor de $20-40 \%{ }^{1-4}$.

Las complicaciones neurológicas secundarias al proceso inflamatorio meníngeo incluyen: alteración del flujo sanguíneo encefálico por aumento de la presión intracraneana (PIC), edema cerebral, hidrocefalia, abscesos cerebrales, convulsiones fo-

Correspondencia a: Dr. Patricio Mellado T. Marcoleta 347, Santiago, Chile. Fax: 56-2-632-6221. E-mail: pmellado@med.puc.cl cales o generalizadas y enfermedad cerebrovascular venosa y arterial, siendo esta última una causa importante que puede explicar el mal pronóstico de las $\mathrm{MBA}^{3-5}$.

Comunicamos el caso de una paciente con una MBA complicada con un infarto maligno de la arteria cerebral media (IMACM) y discutimos algunos aspectos de esta enfermedad.

\section{CASO CLÍNICO}

Mujer de 68 años, diestra, sin antecedentes mórbidos de importancia. Con una historia clínica de dos días de cefalea, vómitos, fiebre y compromiso de conciencia progresivo, ingresó al hospital febril $\left(37,5^{\circ} \mathrm{C}\right)$, 
presión arterial de 150/80 mmHg, examen cardiopulmonar y abdominal normal. Al examen neurológico destacaba sopor superficial y discreta rigidez de nuca, sin déficit focal motor ni sensitivo y fondo de ojo normal. Se planteó el diagnóstico de una MBA y se realizó una tomografía computada (TC) de encéfalo sin contraste, que fue normal (Figura 1) y una punción lumbar. El análisis citoquímico del líquido cefalorraquídeo (LCR) informó: leucocitos= $10 \mathrm{cel} / \mathrm{mm}^{3}$, proteínas $=1,58 \mathrm{~g} / \mathrm{dl}$, glucosa $=0 \mathrm{~g} / \mathrm{dl}$. La tinción de Gram del LCR demostró diplococos Gram positivos. Se hospitalizó en cuidados intermedios de neurología y se inició ceftriaxona $2 \mathrm{~g}$ cada $12 \mathrm{~h}$ iv, asociado a dexametasona $10 \mathrm{mg}$ cada $6 \mathrm{~h}$ iv. Evolucionó con empeoramiento del nivel de conciencia, por lo que a las $24 \mathrm{~h}$ de ingreso se realizó una TC de encéfalo de control, que no mostró cambios en relación a la de ingreso. A las $48 \mathrm{~h}$ del ingreso presentó una convulsión tónico clónica generalizada y se cargó con 1.200 mg de fenitoína oral, seguidos de $200 \mathrm{mg}$ cada $12 \mathrm{~h}$ vo. El cultivo de LCR fue positivo para Streptococcus pneumoniae sensible a la ceftriaxona. Evolucionó en sopor profundo con midriasis izquierda arrefléctica, hemiparesia braquio crural derecha y Babinski derecho. Se realizó una TC de encéfalo a las $72 \mathrm{~h}$ del ingreso

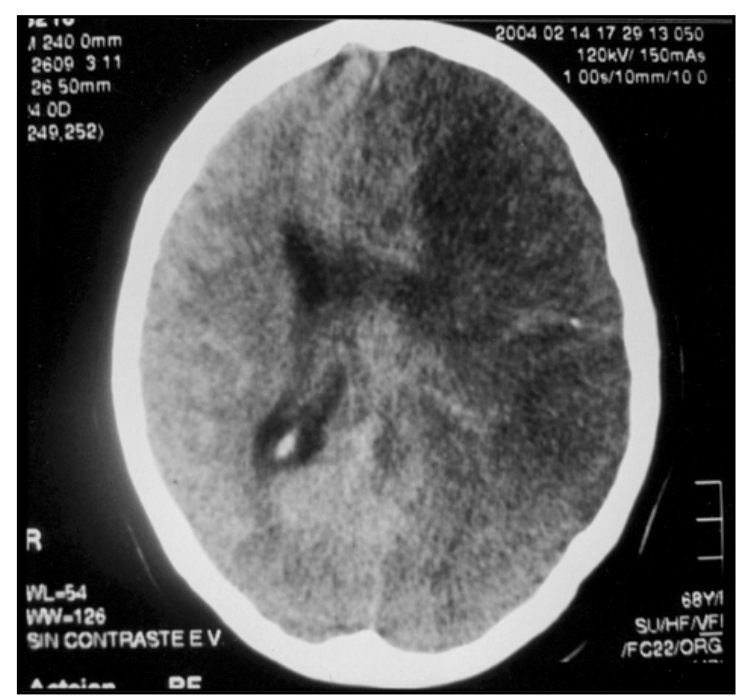

FiguRA 3. Tomografía computada al tercer día a nivel de la corona radiada. Muestra una hipodensidad en el territorio de la arteria cerebral media izquierda compatible con un infarto completo del territorio de esta arteria. que evidenció un infarto completo del territorio de la arteria cerebral media (ACM) izquierda y una hernia uncal ipsilateral (Figuras 2 y 3), falleciendo al 4⿳o día después del ingreso.

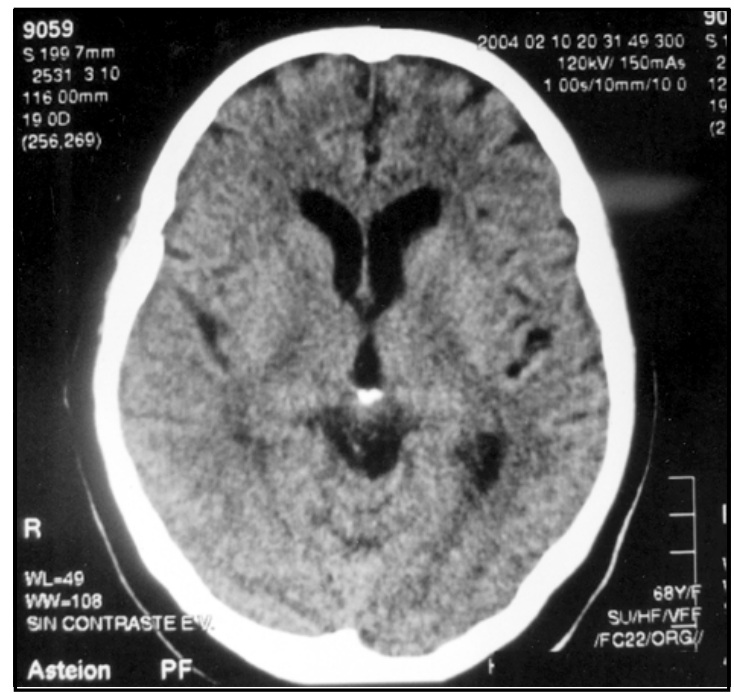

FiguRa 1. Tomografía computada de encéfalo al ingreso, normal. Corte a nivel de la glándula pineal.

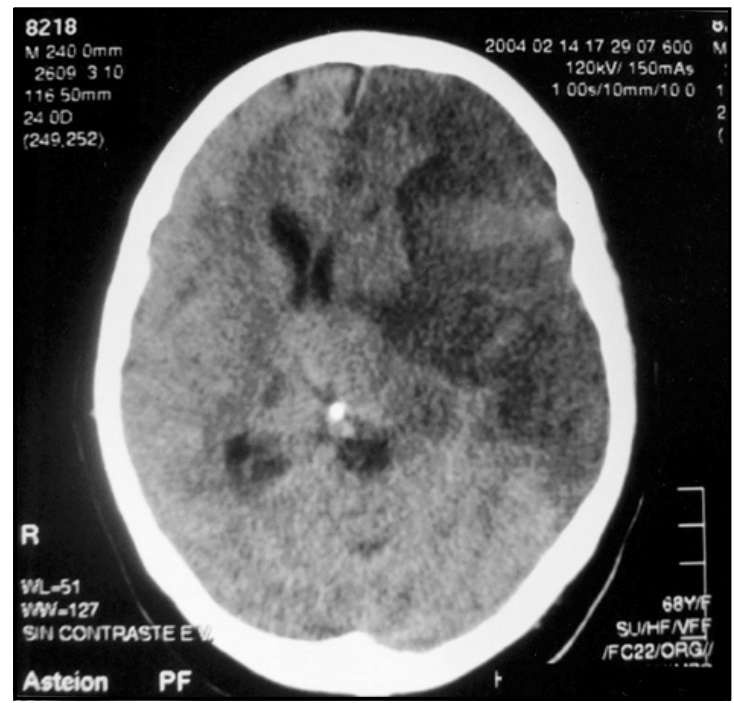

FiguRa 2. Tomografía computada al tercer día a nivel de la glándula pineal. Muestra una hipodensidad en el territorio de la arteria cerebral media izquierda compatible con un infarto completo del territorio de esta arteria. 


\section{DisCUSIÓN}

El compromiso vascular arterial en la MBA se estima sobre $15-20 \% 2,3$, principalmente durante la primera semana de evolución ${ }^{3}$, y ha sido demostrado en estudios patológicos, angiográficos, sonográficos y de perfusión cerebral ${ }^{6-9}$. Se caracteriza por la reducción del diámetro de las grandes arterias basales, probablemente por vasoespasmo, ya que no se han demostrado cambios neuropatológicos compatibles con inflamación ${ }^{10}{ }^{10}$ y por alteración del diámetro de las arterias de mediano y pequeño calibre y de las arteriolas debido a vasculitis secundaria a la extensión de la inflamación a través de los espacios de Virchow-Robin ${ }^{6,10}$. Por otro lado, la elevación de la PIC por edema citotóxico disminuye la perfusión cerebral y puede determinar además la aparición de infartos encefálicos en zonas limítrofes ${ }^{11}$.

En la angiografía cerebral se demuestran alteraciones focales en los vasos de mediano y gran calibre, lo que se evidencia como múltiples estenosis en los primeros segmentos de las arterias intracraneanas y especialmente del segmento supraclinoideo de la arteria carótida interna ${ }^{12}$. Un estudio angiográfico de pacientes con MBA complicada mostró compromiso vascular en $33 \%$ de los $\operatorname{casos}^{13}$. Un estudio de angiografía por resonancia magnética en niños, mostró vasculitis en 3 de 5 pacientes con MBA, y las secuencias de difusión evidenciaron infartos múltiples, principalmente frontales, en 12 de 13 niños ${ }^{14}$.

La ecografía Doppler transcraneana evalúa de forma no invasiva la velocidad del flujo sanguíneo cerebral (VF). El aumento de la VF está determinado principalmente por cambios focales leves del diámetro de vasos de mediano y gran calibre y alteraciones generalizadas a nivel de arteriolas y capilares $^{6}$. Se han demostrado elevaciones leves de la VF en $50 \%$ de los casos y graves en $32 \%$ de los pacientes con $\mathrm{MBA}^{6}$.

El empleo del HMPAO-SPECT puede detectar cambios en la perfusión focal por compromiso de vasos de pequeño calibre. Se ha reportado hipoperfusión encefálica en $93 \%$ de los pacientes con $\mathrm{MBA}^{15}$.
El compromiso vascular en la MBA se ha asociado a una evolución tórpida y a un desenlace fatal $^{16}$. El deterioro de la circulación posterior es menos grave y clínicamente mejor tolerado que los cambios hemodinámicos de la circulación anterior ${ }^{5,12,17}$

En el caso descrito, la paciente presentó un infarto completo de la ACM izquierda, lo que se denomina infarto maligno de la arteria cerebral media (IMACM). El IMACM corresponde al infarto de al menos $2 / 3$ de la ACM y corresponde de 5 a $10 \%$ del total de infartos encefálicos $^{18}$. El gran edema cerebral que acompaña a los IMACM determina la aparición de hernias subfalcina, uncal y diencefálica, cuya progresión se manifiesta clínicamente como un deterioro rostrocaudal de las funciones neurológicas, finalizando con la muerte cerebral $^{19}$. El IMACM tratado médicamente tiene una mortalidad de hasta $80 \% 18$. El tratamiento quirúrgico con una hemicraniectomía descompresiva (HD) en pacientes seleccionados ha mostrado, en trabajos europeos y en la experiencia de uno de los autores, la reducción de la mortalidad, la que ha sido valorada hasta en $12 \%$ si se realiza al momento del ingreso y es guiada por los hallazgos de la resonancia magnética ${ }^{19,20}$. La HD no ha sido descrita en pacientes con IMACM asociado a MBA, en esta paciente se descartó este procedimiento debido a su edad avanzada y por el compromiso del hemisferio dominante.

En relación con el manejo de los infartos encefálicos en el curso de una MBA se ha sugerido evaluar con ensayos clínicos controlados, el uso de terapia hipervolémica e hipertensiva para asegurar el flujo sanguíneo cerebral y evitar las complicaciones del vasoespasmo asociado a la $\mathrm{MBA}^{5}$.

En conclusión: El compromiso vascular arterial en el contexto de una MBA es frecuente y explica en un grupo de estos pacientes su alta morbimortalidad. Los estudios angiográficos y patológicos muestran vasoespasmo en las arterias de gran calibre y vasculitis en las de mediano y pequeño calibre. 


\section{REFERENCIAS}

1. TunKel AR. Bacterial Meningitis. Philadelphia. Lippincott Williams and Wilkins. 2001.

2. Kastenbauer S, PFister H. Pneumococcal meningitis in adults. Spectrum of complications and prognostic factors in a series of 87 cases. Brain 2003; 126: 1015-25.

3. Pfister HW, Feiden W, Einhäupl KM. Spectrum of complications during bacterial meningitis in adults. Arch Neurol 1993; 50: 575-81.

4. Del BRutto OH. Infections and stroke. En: Ginsberg MD, Bogousslavsky J (eds). Cerebrovascular Disease. Pathophysiology, diagnosis and management. Malden. Blackwell Science 1998; 1628-46.

5. Mü山er M, Merkelbach S, Hermes M, König J, SchimrigK K. Relationship between short term outcome and occurrence of cerebral artery stenosis in survivors of bacterial meningitis. J Neurol 1998; 245: 87-92.

6. Rues S, Schminke U, Fassbender K, DafFertshofer M, SteinKe W, Hennerici M. Cerebrovascular involvement in the acute phase of bacterial meningitis. J Neurol 1997; 244: 51-5.

7. Mukai K, Yasuda T, Hara K, Funakawa I, Terao A. Adult pneumococcal meningitis complicated by cerebral infarction: a case report (abstract). Rinsho Sinkeigaku 1996; 36: 1110-13.

8. Perry JR, Bilbao JM, Gray T. Fatal basilar vasculopathy complicating bacterial meningitis. Stroke 1992; 23: 1175-8.

9. Hsu S, KIM H. Meningococcal meningitis presenting as stroke in an afebrile adult (report). Ann Emerg Med 1998; 32: 620-3.

10. Vernino S, Wijdicks E, McGough PF. Coma in fulminant pneumococcal meningitis: New MRI observations. Neurology 1998; 51: 1200-2.

11. TAKEOKA M, TAKAHASHI T. Infectious and inflammatory disorders of the circulatory system and stroke in childhood. Curr Opin Neurol 2002; 15: 159-64.

12. Palacio S, Hart R, Vowmer D, Kagan-Hawet K. Late developing cerebral arteriopathy after pyogenic meningitis. Arch Neurol 2003; 60: 431-3.

13. Pfister HW, Borasio GD, Dinagl U, Bauer M, EINHÄUPL KM. Cerebrovascular complications of bacterial meningitis in adults. Neurology 1992; 42: 1497-504.

14. Jan W, Zimmerman RA, Bilaniuk LT, Hunter JV, Simon EM, Haselgrove J. Diffusion weighted imaging in acute bacterial meningitis in infancy. Neuroradiol 2003; 45: 634-9.

15. FörderReuther S, Tatsch K, EinhäUpl KM, Pfister HW. Abnormalities of cerebral blood flow in the acute phase of bacterial meningitis in adults. J Neurol 1992; 239: 431-6.

16. Mü山er M, Merkelbach S, Huss GP, Schimrigk K. Clinical relevance and frequency of transient stenoses of the middle and anterior cerebral arteries in bacterial meningitis. Stroke 1995; 26: 1399-403.

17. MüLer M, Merkelbach S, Schimrigk K. Cerebral hemodynamics in the posterior circulation of patients with bacterial meningitis. Act Neurol Scan 1996; 93: 443-9.

18. Hacke W, Schwab S, Horn M, Spranger M, De Georgia M, Von Kummer R. The «malignant»middle cerebral artery territory infarction: clinical course and prognostic signs. Arch Neurol 1996; 53: 30915.

19. Melado P, Romero C. Infarto maligno de la arteria cerebral media. En: Castillo L, Romero C, Mellado P (eds). Cuidados Intensivos Neurológicos. Santiago: Mediterráneo, 2004; 275-85.

20. Georgiadis D, Schwarz S, Aschoff A, Schwab S. Hemicraniectomy and moderate hypothermia in patients with severe ischemic stroke. Stroke 2002; 33: 1584-8. 